Fecha de recepción: abril 2020 Fecha de aceptación: mayo 2020 Versión final: junio 2020

\section{La historia y el Diseño Industrial en la Universidad Rafael Landívar. Breve descripción de la génesis, la investigación y la apertura hacia la tecnología de impresión 3D}

Hernán Ovidio Morales Calderón ${ }^{(1)}$

\begin{abstract}
Resumen: En 1987, el entonces rector de la Universidad Rafael Landívar (URL), Luis Manresa Formosa, S. J., dirigió la formulación y apertura de la carrera de Diseño Industrial, con una orientación hacia el fortalecimiento de los sectores artesanales y agrícolas de Guatemala. Luego en el año 2000, se presenta una oportunidad para dar un paso hacia la investigación en diseño, abriendo las puertas del Instituto de Investigación y estudios Superiores en Arquitectura y Diseño (INDIS).

El presente artículo presenta la evolución de la academia landivariana, desde su interés por el sector artesanal y agrícola (vocación y sello diferenciador aún presente en la actualidad) la investigación formal y su evolución de la mano con las nuevas tecnologías de fabricación digital.

La URL para ampliar y generar un mayor impacto, ha implementado desde el INDIS el "Centro de Investigación, Capacitación y Diseño en Tecnologías Alternativas (CTA)" con el fin de fortalecer el vínculo de la investigación y la academia, promover el acceso a la tecnología a diversos sectores y democratizar la tecnología de impresión 3D, acercando a la población a sistemas actualizados y de vanguardia.

Es así como el CTA del INDIS, dentro del esquema de la Vicerrectoría de Investigación y Proyección (VRIP) desarrolla proyectos de investigación, formación e incidencia, además de brindar servicios de impresión y asesoría a externos para el desarrollo de la industria guatemalteca.
\end{abstract}

Palabras clave: Historia - Investigación - Acción - Diseño Industrial - Fabricación digital - Impresión 3D - Tecnologías Alternativas.

[Resúmenes en inglés y portugués en la página 132]

(1) Doctor en Diseño (Universidad de Palermo, Argentina). Maestría en Docencia de la Educación Superior (Universidad Rafael Landívar). Maestría en Diseño Industrial (ISTHMUS, Panamá) Licenciatura en Diseño Industrial (Universidad Rafael Landívar). Director del Instituto de Investigación y Estudios Superiores en Arquitectura y Diseño (INDIS) de la Universidad Rafael Landívar, Guatemala. 


\section{Introducción}

Diferentes autores han escrito sobre la historia del diseño industrial en el mundo, para éste artículo será importante un breve recorrido histórico, abordando principalmente desde el Arts and Crafts hasta la Hfg. Lo anterior, para dar pie a los referentes que influenciaron al diseño en América Latina y especialmente los que sirvieron de modelo a la academia guatemalteca para la implementación del Diseño Industrial.

El campo disciplinar del Diseño Industrial está presente en Guatemala desde hace más de treinta años. Fue la Universidad Rafael Landívar (URL), por medio de su Facultad de Arquitectura y Diseño, la primera universidad en el país que incorporó esta carrera con el fin de diversificar la oferta académica y, como una oportunidad para el desarrollo de los sectores agrícola y artesanal. El programa inició en el año 1987 con un nivel de titulación de técnico universitario. La primera cohorte egresó a finales de 1990 y en 1995, luego de una renovación curricular, se dio inicio al programa de licenciatura.

Todo esto, ocurrió gracias al interés del entonces Rector, Monseñor Luis Manresa Formosa, quién con su experiencia de trabajo durante su gestión como Obispo de los Altos en el occidente del país, encontró una alternativa viable en el diseño. Su apoyo principal, el Arquitecto Daniel Borja, operó todas las indicaciones administrativas necesarias para conseguir la incorporación de la carrera a la URL.

El diseño industrial fue fortaleciendo su presencia, hasta que en el año 2000, se presenta un importante hito en la historia de la disciplina: el cambio de nombre de la facultad, de Facultad de Arquitectura a Arquitectura y Diseño, además de la formalización de la investigación y proyección de las disciplinas proyectuales.

Es así como nace el Instituto de Investigación y Proyección Social INDIS que luego cambiaría de nombre con la reestructuración de la Vicerrectoría de Investigación y Proyección (VRIP).

Es entonces como luego de éste breve recorrido histórico, el diseño industrial llega a un punto de inflexión importante, presentando estructuras de investigación fortalecidas que además incorporan la proyección universitaria. La investigación formativa es articulada directamente con la docencia y desde entonces se ha sistematizado una fórmula para atender problemas del entorno con presencia landivariana.

Los procesos de modernización no fueron ajenos al diseño guatemalteco. Con el pasar del tiempo se fueron implementando espacios como el laboratorio de modelos y prototipos, incorporando nuevos equipos y tecnologías. El más reciente, el Centro de Investigación, Capacitación y Diseño en Tecnologías Alternativas, espacio que atiende solicitudes al interno y al externo de impresión 3D.

El presente artículo presenta la evolución del diseño en la historia hasta llegar a la academia landivariana, su interés por el sector artesanal y agrícola (vocación y sello diferenciador aún presente en la actualidad) la investigación formal y su evolución de la mano con las nuevas tecnologías de fabricación digital. 


\section{Sobre la historia}

Nietzsche definió tres maneras de estudiar y escribir la historia (Fernández y Bonsiepe, 2008, pp. 12-13): la historia monumental, la historia anticuaria y la historia crítica:

La historia monumental proporciona ejemplos, puntos de referencia, sobre todo en tiempo oscuros, en los cuales ella mantiene "la creencia en la humanidad". A la historia anticuaria, Nietzsche la caracteriza de la siguiente manera: "La Historia, por lo tanto, pertenece además a quien conserva y venera, a quien contempla con fidelidad y con amor el lugar del que viene y por el que es lo que es". Y por fin el tercer modo de considerar el pasado, la historia crítica: "El (Hombre) debe tener la fuerza, y de vez en cuando aplicarla, de quebrar un pasado y disolverlo para poder vivir. Él logra esto al arrastrarlo al tribunal, en cuestionarlo penosamente y por fin en sentenciarlo (...) todo pasado merece ser sentenciado".

Nietzsche no consideraba la historia solo como una línea temporal de acontecimientos pasados, sino que siempre relacionaba esos acontecimientos con el tiempo presente.

La historia del diseño (así como las de otras ramas del saber) no debe ser vista como una mera enumeración de datos cronológicos, sino que estos deben ponerse en contexto para ser una variable de los procesos socioeconómicos y sociopolíticos.

$\mathrm{Al}$ estudiar el pasado, la historia ayuda a comprender el presente y a establecer una comunicación con las generaciones anteriores, ya que se escucha su música, se leen sus textos, se aprecian sus obras de arte, se ven sus fotografías, etc. (Campi, 2013): "Leibnitz incluía, entre los beneficios de la historia 'los orígenes de las cosas presentes descubiertos en las cosas pasadas, porque una realidad no se conoce nunca mejor que por sus causas"' (Campi, 2013, p. 22).

\section{Breve recorrido de la historia del diseño}

Este relato inicia con Arts and Crafts (su premisa fue lo artesanal, el rechazo a la máquina), el movimiento trajo un renacimiento de la artesanía, pero no del diseño a la industria, ya que rechazaba tanto la modernidad como la industrialización. No promovió un estilo específico, sino que se centró en darle importancia al artesano, quien estaba siendo sustituido por las máquinas. A finales del siglo XIX, durante las décadas de 1880 y 1890, surgió la segunda generación del Arts and Crafts, que se caracterizó, entre otros, por aceptar el uso de las máquinas, pero como auxiliares de los artesanos.

Le sigue en Europa y América desde la última década del siglo XIX hasta 1914 el Art Nouveau (la curvilínea, el equilibrio entre la funcionalidad y la belleza). A diferencia del Arts and Crafts, el Art Nouveau sí hizo uso de las máquinas, pero siempre «buscó restaurar el equilibrio entre las artes y los oficios y desarrolló al máximo las técnicas artesanales» 
(Gay y Samar, 2004, p. 63). Se esforzó por combinar y guardar el equilibrio entre la funcionalidad y la belleza de los objetos de uso cotidiano que creó.

El siguiente movimiento, Werkbund (lo funcional, lo utilitario, lo objetivo) tuvo como objetivo agrupar artesanos, artistas, arquitectos, diseñadores, etc. para encontrar la manera de crear, en serie y con alta calidad, productos industriales de consumo diario.

Sigue la Bauhaus ${ }^{1}$ que surgió en 1919 a raíz de la fusión de la Escuela Superior de Bellas Artes y la Escuela de Artes Aplicadas. La Bauhaus “... es reconocida como la primera escuela de diseño industrial a nivel mundial [...] incluso ha sido mitificada y emulada con el afán de obtener diseñadores sobresalientes. Sin embargo [...] estuvo condicionada por un tiempo y un lugar...” (Salinas, 2003, p. 109).

Tuvo tres períodos importantes, que corresponden al lugar donde se desarrolló: Weimar (1919-1925), Dessau (1925-1932), Berlín (1932-1933). La estética de los productos originados durante este período estuvo basada en "formas originadas en ideas abstractas, basadas en leyes matemáticas y en la geometría euclidiana cuya materialización se sintetiza en tres aspectos: uniformidad, estandarización y rigurosidad estético-expresiva" (Gay y Samar, 2004, p. 109).

Para finalizar el corto recorrido histórico, concluye la Hochschule für Gestaltung o Hfg (estética funcionalista, incorporación de conocimientos científicos al diseño, compromiso social del diseño). Fue una institución universitaria privada establecida en Ulm, Alemania, en 1955. Fue iniciativa de las hermanas Inge y Gretel Scholl y de Otl Aicher -esposo de la primera- a través de la Fundación Hermanos Scholl. Esta entidad fue creada por ellos en memoria Hans y Sophie Scholl -hermanos de Inge y Gretel-, quienes fueron ejecutados en 1943 por el partido nazi.

La institución llevó a cabo sus actividades en un edificio construido por el arquitecto suizo Max Bill, quien fue también el primer rector de la escuela y quien "...había sido hasta el momento el principal exponente del movimiento de la Gute Form (Buena Forma), que pugnaba por una nueva postura en el diseño contraria al Styling" (Salinas, 2003, p. 170). Aunque Bill la consideró como una continuación de la Bauhaus, y Gropius autorizó el uso del nombre "Bauhaus Ulm", los fundadores de la institución conservaron el nombre original: Hochschule für Gestaltung. La HfG nunca renegó de la Bauhaus; simplemente, trató de adaptarse a los avances tecnológicos de la época y crear conforme a ello. El principal aporte fue formar "diseñadores para la industria de los bienes de consumo y de producción tanto como para los modernos medios de comunicación, impresos, cinematógrafo, radio y publicidad. [...]. Al mismo tiempo, deben tomar en cuenta las consecuencias culturales y sociales de su trabajo" (Gay y Samar, 2004, p. 142).

Hasta ahora, se ha abordado el concepto de historia que da fundamento al artículo (conociendo el contexto en el cual surge el diseño) y un esbozo de la evolución del diseño en el devenir del tiempo. Se enlaza a continuación con el referente que tuvo América Latina para incorporar la disciplina en la oferta académica, Lo anterior, para después caracterizar las condiciones sociales y económicas como escenario para el surgimiento del Diseño Industrial. 


\section{La impronta ulmiana en el continente}

La influencia de la Escuela de Ulm en América Latina ha sido de notable envergadura: durante las décadas de los sesenta y de los setenta, las políticas económicas en América Latina tendieron a sustituir las importaciones y desarrollar la industria nacional. Sin embargo, desde la década de los ochenta, “... [América Latina] fue arrastrada a la globalización y a políticas de endeudamiento que generaron una nueva forma de dependencia" (Fernández, 2002, p. 39). Además, es en esas décadas -sesenta y setenta- que empiezan a proliferar las escuelas de diseño en la región. Es en este contexto que la Ulm adquiere importancia en la región latinoamericana, y es en el campo académico, más que industrial, donde está arraigado el diseño en Latinoamérica, así como en el sector privado, “...evidenciándose la falta de incidencia en el entorno social” (Fernández, 2002, p. 66). Son Brasil, Argentina, Chile, México, Cuba, Colombia, Venezuela y Perú los países que inician la enseñanza del diseño durante la década de los sesenta; lo hacen tomando como directriz las bases de la Escuela de Ulm:

El programa y los valores de la hfG Ulm fueron los que más influencia marcaron en el origen del diseño en el continente, principalmente en los países donde la conciencia de diseño como factor de la economía fue más notoria, donde la clase política demostró sensibilización y conocimiento de la importancia del diseño en el proceso de industrialización y comercialización, se lograron los niveles más altos de desarrollo de la actividad (Fernández, 2002, p. 63).

Cabe decir que durante la década de los sesenta y la primera mitad de los setenta, en América Latina el diseño estuvo más avanzado que en muchos países de Europa, tanto en el campo profesional como en el de la enseñanza (Fernández y Bonsiepe, 2008).

\section{El contexto de Guatemala hacia el progreso: industrialización y tecnificación}

Los procesos de modernización en Guatemala estuvieron marcados por la Ley de Fomento Industrial (Decreto 459), aprobada en 1947, la cual declaraba, como de urgencia nacional, el desarrollo de industrias que aprovecharan los recursos del país; esto fue nuevo, ya que el país había sido, hasta entonces, eminentemente agrícola. El artículo 1 de la Ley de Reforma Agraria benefició el crecimiento industrial en diferentes sectores del país; su objetivo fue "liquidar la propiedad feudal en el campo y las relaciones de producción que la originan para desarrollar la forma de explotación y métodos capitalistas de producción en la agricultura y preparar el camino para la industrialización de Guatemala (Guerra Borges, 2011, p. 50). Tras la Segunda Guerra Mundial, luego de que Latinoamérica sufrió las consecuencias de no poder importar productos ni materia prima debido justamente a esta y a la Primera Guerra Mundial, entre los años 1950 y 1973 surgieron industrias en sustitución de las importaciones, donde creció fuertemente el comercio entre países de América Latina: 
En la década de 1960 se entró de lleno a la industrialización. Esto ha sido el cambio cualitativo más importante de la economía guatemalteca en los últimos sesenta años... principalmente mediante el mecanismo del sacrificio fiscal (exención de impuestos), pero también mediante grandes inversiones en infraestructura (Guerra Borges, 2011, p. 71).

Frente a los cambios en los mercados internacionales, se realizaron esfuerzos en el área de tecnificación del cultivo del café y de la caña de azúcar, ya que tanto el café como el azúcar eran los productos de mayor exportación desde Guatemala:

El proceso de modernización tecnológica del cultivo de la caña y la producción de azúcar son más intensos y variados que los del café; incluyen elementos como los siguientes: integración de ingenios azucareros y de fincas mediante la adquisición o arrendamiento de tierras y la generación de la energía que consumen; nuevas variedades de caña, nuevos métodos de cultivo, control de plagas, introducción del riego, uso de un nuevo tipo de machete conocido como "australiano", mecanización y aumento de la capacidad de carga y la introducción de nuevas centrífugas (Guerra Borges, 2011, p. 97).

Según Guerra Borges (2011), la industrialización sustitutiva provocó un fuerte dinamismo, creando un nuevo sector, fuentes de empleo y mejor calidad en la oferta de los productos ofrecidos, en contraste con el sector agrícola. La inversión se orientó a las industrias tradicionales, debido al capital y a la tecnología como oportunidades de producción. Algo que benefició fuertemente a la industria, fueron los bajos aranceles de importación en materias primas.

\section{La llegada del diseño industrial y su puesta en marcha}

El diseño industrial en Guatemala como carrera universitaria, no solo nace en un ambiente de movimientos de diseño, sino también influenciado por las situaciones sociales y económicas particulares del país abordadas en el título anterior.

Se pueden definir tres épocas en la historia del diseño en la URL. La primera época transcurrió de 1975 a 1986, cuando un grupo de arquitectos formuló la carrera técnica Diseño de Productos (Diseño Industrial), plan de estudio que fue aprobado por el Consejo Directivo de la URL, pero que no contó con estudiantes. La segunda época se desarrolló entre 1986 y 1987, cuando la Compañía de Jesús notó, en el diseño industrial, posibilidades para promover el desarrollo de los sectores artesanal y agrícola. Finalmente, la tercera época, que transcurre de 1987 a 2017, con la puesta en marcha de la formación de los diseñadores industriales guatemaltecos (Morales, 2019).

La Universidad Rafael Landívar (URL), opta por un modelo educativo que detecta una carencia del diseño en dos sectores importantes, el artesanal y el agroindustrial.

Lo anterior se define en el documento propuesto del programa de Diseño Industrial de 1986, que el perfil del diseñador está basado en el contexto de la época, que conllevaba 
interpretar las necesidades respecto a la cultura y al país para materializar los aportes que existían o que estaban próximos a existir. Se perfilaba como un profesional que aplicaría sus conocimientos, recursos y tecnologías para utilizar el diseño como servicio con el único fin de proveer desarrollo comunitario y social, dejando a un lado los intereses mercantilistas (Borja, Contreras y Rivera, 1986, p. 21).

El equipo que estuvo a cargo de la conformación del proyecto de Diseño Industrial, detectó dos situaciones en la fase de diagnóstico de la problemática: “a) Escaso e inadecuado desarrollo artesanal, b) Falta de incentivos en el área de Diseño para el Desarrollo Industrial y Agro-industrial" (Borja, Contreras y Rivera, 1986).

El 26 de septiembre de 1986 la Facultad de Arquitectura entregó a los Consejos Ejecutivo y Directivo de la URL el proyecto de la carrera de Diseño Industrial, elaborado por la comisión de estudio de Diseño Industrial, conformado por el arquitecto Daniel Borja, el ingeniero José Antonio Contreras Godoy y el diseñador industrial Sergio Rivera Conde (éste último asesor, afiliado a la Universidad Autónoma Metropolitana, Unidad Xochimilco; institución de educación superior referente y que marca la influencia en el diseño que iniciaba en Guatemala). La carrera Técnica de Diseño Industrial fue aprobada el 18 de febrero de 1987 por el Consejo Directivo.

Esta particularidad determinó y distinguió la institucionalización del diseño industrial en Guatemala como carrera universitaria, la cual tuvo lugar en 1986 por la instrucción directa del Rector Manresa, quien le encomendó a Daniel Borja preparar las bases para poner en marcha la carrera de Diseño Industrial.

\section{La investigación y proyección: nacimiento del INDIS}

La apuesta institucional por fortalecer la investigación formal y la proyección social en la disciplinas de la arquitectura el diseño en la universidad Rafael Landívar (URL) se remonta al año 2000. Es en el Acta número 10-2000 con fecha trece de octubre del año dos mil, donde el Consejo Directivo aprueba la creación del Instituto de Investigación y Proyección Social (INDIS), iniciando sus labores en enero del 2001. El Instituto opera originalmente en la Facultad de Arquitectura y Diseño, como una iniciativa dentro del proceso de renovación curricular para responder a la necesidad de contar con una unidad encargada del manejo de proyectos y la investigación de la Facultad de Arquitectura y Diseño. El nombre inicial adoptado era el de Instituto de Investigación y Proyección Social.

Es con la creación de la Dirección de Investigación -ahora Vicerrectoría de Investigación y Proyección (VRIP), que el nueve de noviembre del dos mil quince de acuerdo a la resolución de Rectoría número 38-15, se aprueba el cambio de nombre a "Instituto de Investigación y Estudios Superiores en Arquitectura y Diseño".

Desde su génesis, el trabajo del INDIS con la Vicerrectoría Académica, específicamente con la Facultad de Arquitectura y Diseño, ha consolidado un modelo para conocer mejor la forma de insertar los problemas de la sociedad a la universidad. En otras palabras, llevar la realidad a la academia, donde ha logrado la generación de conocimiento, el desarrollo 
de respuestas integrales y además, la participación y acompañamiento del instituto en investigación acción.

Desde el INDIS se desarrollan programas de investigación e incidencia en concordancia con la Agenda de Investigación y Proyección (AIP) de la VRIP. Dentro de sus programas se encuentra el Centro de Investigación, Capacitación y Diseño en Tecnologías Alternativas (CTA), para brindar soluciones de diseño por medio de la implementación de tecnologías alternativas en el país.

En el año 2018, con la aprobación del proyecto de Planificación Operativa Anual -POAde la Vicerrectoría de Investigación y Proyección (VRIP) como apoyo al Plan Estratégico de la Universidad, se disponen de los recursos para el equipamiento del -en ese entonces denominado- Centro de capacitación tecnologías alternativas (CTA).

Como resultado, en colaboración con la Facultad de Arquitectura y Diseño 33 estudiantes de la licenciatura en Diseño Industrial del curso Proyecto 5 (bajo la figura de consultores junior e investigadores asociados del INDIS), en el 2018 se presentaron seis proyectos de tecnología alternativa orientados al sector agrícola guatemalteco.

Ese mismo año, y con los buenos resultados obtenidos con la experiencia del proyecto, el equipo de Rectoría Task Force de inversiones, analiza y aprueba la propuesta del INDIS de la expansión del CTA tanto en espacio físico como en equipaminto. En su segundo año de operación (2019) se implementa el proyecto Laboratorio de investigación, capacitación y diseño en tecnologías alternativas, con la adquisición de 11 máquinas de impresión 3D y la infraestructura adecuada para el laboratorio tanto en arquitectura como mobiliario.

El CTA ofrece servicios tecnológicos de impresión 3D y generación de tecnologías alternativas a la comunidad landivariana y a sectores públicos y privados. Dispone de infraestructura y equipo para facilitar y democratizar la tecnología con capacidad de atender a 15 usuarios simultáneamente.

El CTA cuenta con 12 estaciones con impresoras 3D con tecnología FDM: Modelado por deposición y fundición (Fused Deposition Modeling) o FFF: Fabricación por función de filamentos fundidos (Fused Filament Fabrication). Las impresoras son de fuente abierta (open source), tienen superficie de impresión con temperatura controlada, calibración automática y capacidad de imprimir un gran rango de materiales (PLA, ABS, Nylon, entre otros).

\section{El INDIS y la lucha contra el Covid-19}

La respuesta del INDIS se hizo a través del CTA en colaboración con el colectivo nacional Makers GT. Desde la URL se acordó apoyar en la impresión de los protectores faciales, en coordinación con otras comisiones de la iniciativa responsables del acopio y la distribución. Gracias a la donación de colaboradores de la universidad, se pudieron adquirir los recursos necesarios para la fabricación de los protectores faciales (filamento de ácido poliácido o PLA, acetato, bandas elásticas, bolsas ziploc y cajas de cartón).

Debido a las disposiciones de cierre de instituciones educativas dictadas por el gobierno de Guatemala, se solicitó un permiso especial para ingresar a las instalaciones del Campus 
Central. Las labores se efectuaron en jornada única, respondiendo también a las disposiciones de toque de queda. Así es como en un horario de 7:00 a 13:00 horas, se trabajó en la producción.

Tres personas integraron el equipo: Director del INDIS, Dr. Ovidio Morales; Coordinador del CTA, DI Ervin Moreno y Coordinador del CTA, DI Rodrigo Mayén.

La comunidad landivariana responde con la donación de 700 protectores faciales para el personal de salud que se enfrenta a la pandemia del Covid-19. En una invitación especial a las instalaciones de la URL dirigida al Presidente de la República, Dr. Alejandro Giammattei, se realizó una entrega simbólica. Los protectores faciales fueron empacados en bolsas de cierre hermético y en cajas de cartón para seguir con las normas de seguridad sanitaria requeridas y así poderlas transportar de mejor manera.

La entrega física de la de donación de protectores faciales se hizo a dos lugares. El 24 de abril al nodo de acopio de Makers GT en Campus TEC (300) y el 27 de abril en el hospital temporal del Parque de la Industria (400).

La Universidad Rafael Landívar, busca espacios de apoyo para la sociedad en estos momentos de crisis, porque somos conscientes del momento que vivimos, es ahora cuando nuestro compromiso se hace más fuerte para salir juntos adelante.

\section{Notas}

1. Bauhaus: palabra del idioma alemán que significa, literalmente, "casa de construcción".

\section{Listado de referencias bibliográficas}

Borja, D.; Contreras, J. y Rivera, S. (1986). Proyecto “Diseño Industrial, Carrera de Nivel Técnico". Anexo A, Acta N. ${ }^{\circ}$ 3-87 del Consejo Directivo de la Universidad Rafael Landívar, 1987.

Campi, I. (2013). La historia y las teorías historiográficas del diseño. México: Editorial Designio.

Fernández, S. y Bonsiepe, G. (2008). Historia del diseño en América Latina y el Caribe. Brasil: Editora Blücher.

Gay, A. y Samar, L. (2004). El diseño industrial en la historia. 2a. edición, aumentada. Argentina: Ediciones Tec.

Guerra Borges, A. (2011). Guatemala: 60 años de historia económica (1944-2004). Guatemala: Fundación Soros - PNUD.

Morales, O. (2019). La institucionalización del Diseño Industrial en Guatemala durante la década de los años 80. Universidad de Palermo. Recuperado de: HYPERLINK "https:// www.palermo.edu/dyc/doctorado_diseno/tesis.html" 5 de junio de 2020

Salinas Flores, O. (2003). Historia del diseño industrial. México: Editorial Trillas. 


\begin{abstract}
In 1987, the then rector of the Rafael Landívar University (URL), Luis Manresa Formosa, S. J., directed the formulation and opening of the Industrial Design career, with an orientation towards strengthening the artisanal and agricultural sectors of Guatemala. Then in 2000, an opportunity presents itself to take a step towards design research, opening the doors of the Institute for Research and Higher Studies in Architecture and Design (INDIS). This article presents the evolution of the landivarian academy, from its interest in the artisanal and agricultural sector (vocation and distinctive mark still present today) formal research and its evolution hand in hand with the new digital manufacturing technologies. The URL to expand and generate a greater impact, has been implemented by INDIS the "Center for Research, Training and Design in Alternative Technologies (CTA)" in order to strengthen the link between research and academia, promoting access to technology to various sectors and democratize $3 \mathrm{D}$ printing technology, bringing the population closer to updated and cutting-edge systems.

This is how the INDIS CTA, within the framework of the Vice-Rectory for Research and Projection (VRIP) develops research, training and advocacy projects, in addition to providing printing and advisory services to outsiders for the development of the Guatemalan industry.
\end{abstract}

Keywords: History - Research - Action - Industrial Design - Digital Manufacturing - 3D Printing - Alternative Technologies.

Resumo: Em 1987, o então reitor da Universidade Rafael Landívar (URL), Luis Manresa Formosa, S.J., dirigiu a formulação e abertura da carreira de Desenho Industrial, com orientação para o fortalecimento dos setores artesanal e agrícola da Guatemala. Então, no ano 2000, surge uma oportunidade de dar um passo em direção à pesquisa em design, abrindo as portas do Instituto de Pesquisa e Estudos Superiores em Arquitetura e Design (INDIS). Este artigo apresenta a evolução da academia landivariana, a partir de seu interesse no setor artesanal e agrícola (vocação e marca distintiva ainda hoje presente) na pesquisa formal e sua evolução de mãos dadas com as novas tecnologias de manufatura digital.

A URL para expandir e gerar um maior impacto foi implementada pelo INDIS, o "Centro de Pesquisa, Treinamento e Design em Tecnologias Alternativas (CTA)", a fim de fortalecer o vínculo entre a pesquisa e a academia, promovendo o acesso a tecnologia para vários setores e democratize a tecnologia de impressão 3D, aproximando a população de sistemas atualizados e de ponta.

É assim que o INDIS CTA, no âmbito da Vice-Reitoria de Pesquisa e Projeção (VRIP), desenvolve projetos de pesquisa, treinamento e advocacia, além de fornecer serviços de impressão e consultoria a pessoas de fora para o desenvolvimento da indústria guatemalteca.

Palavras chave: História - Pesquisa - Ação - Design Industrial - Manufatura Digital - Impressão 3D - Tecnologias Alternativas.

[Las traducciones de los abstracts fueron supervisadas por el autor de cada artículo] 